\title{
Producción científica sobre fotoprotección en menores de 14 años de países latinoamericanos: Un estudio bibliométrico.
}

Scientific production on photoprotection in children under 14 from Latin American countries: A bibliometric study.

Diaz-Morales Claudia E.,a

1. Segunda Especialidad de la Facultad de Medicina. Universidad Nacional San Luis Gonzaga de Ica, Peru

a. Residente del Pediatría - Hospital Regional de Ica

\section{Correspondencia:}

Claudia E. Diaz Morales

Número de celular: 956747941

Correo Electrónico:

clau_dm182@hotmail.com

Domicilio: Calle Fermín Tangüis

J-152 Urb. San Miguel -Ica, Peru.

Contribuciones De Autoría:

CEDM : Contribuciones en la concepción y diseño del manuscrito, recolección, análisis e interpretación de los datos, redacción y revisión crítica del contenido del manuscrito y aprobación final del artículo.

Conflicto De Intereses: No declarados.

Financiamiento: Autofinanciado.

\section{Como Citar}

Diaz Morales Claudia E. Producción científica sobre fotoprotección en menores de 14 años de países latinoamericanos: Un estudio bibliométrico. Rev méd panacea 2018; (3): 97-100

Recibido: 26 - 09 - 2018

Aceptado: 30 - 11 - 2018

Publicado: 28 - 12 - 2018

\section{RESUMEN}

Objetivo: Identificar la producción científica sobre nivel de conocimientos, actitudes y prácticas sobre fotoprotección en padres de menores de 14 años latinoamericanos. Materiales y métodos: Estudio bibliométrico descriptivo y transversal. Se realizó una búsqueda bibliográfica en las bases de datos: PubMed, Scopus y la Biblioteca Virtual de Salud, utilizando las palabras claves: "fotoprotección" "niños", "escolares", entre el primero de enero del 2013 al 31 de junio del 2018. Se excluyeron artículos no originales o que no midieran conocimiento sobre fotoprotección. Resultado: Se encontró un total de seis artículos el $28.3 \%$ de los padres reportaron que sus hijos se exponen más de 60 minutos al sol entre semana; en el fin de semana se incrementa a un $42.8 \%$. En Chile, el $14.2 \%$ de los niños han presentado alguna vez quemadura solar, con $2 / 3$ de la población de estudio pasan más de 2 horas diarias al exterior. En Uruguay, El $55 \%$ de los padres afirmó que se protegen del sol, el $30 \%$ solo a veces y el $15 \%$ no se protege. En Cuba el $72.5 \%$ han presentado al menos una quemadura solar. En Perú, El estudio peruano demostró que el $48 \%$ utiliza protector solar en crema, el $46 \%$ ropa de protección y el $51 \%$ gorro o sombrero. Conclusiones: Se requieren intervenciones sanitarias para incrementar el conocimiento de los padres sobre fotoprotección, en México demuestra que una intervención educativa puede incrementar en un futuro el conocimiento de los jóvenes en el cuidado de la piel.

Palabras clave: Medidas de fotoprotección, fotoprotección en niños, efectos de la radiación.

\begin{abstract}
Objective: To identify scientific production on the level of knowledge, attitudes, and practices on photoprotection in parents of children under 14 years of age in Latin America. Materials and methods: Descriptive and transversal bibliometric study. A bibliographic search was carried out in the databases: PubMed, Scopus and the Virtual Health Library, using the keywords: "photoprotection" "children", "school children", between January 1, 2013, and June 31, 2018, We excluded articles that were not original or that did not measure knowledge about photoprotection. Result: A total of six articles were found, $28.3 \%$ of parents reported that their children are exposed to more than 60 minutes in the sun during the week; in the weekend it increases to $42.8 \%$. In Chile, $14.2 \%$ of children have ever presented sunburn, with $2 / 3$ of the study population spending more than 2 hours a day abroad. In Uruguay, 55\% of parents said they protect themselves from the sun, $30 \%$ only sometimes and $15 \%$ do not protect themselves. In Cuba, $72.5 \%$ have presented at least one sunburn. In Peru, the Peruvian study showed that $48 \%$ use sunscreen cream, $46 \%$ protective clothing and $51 \%$ hat or hat. Conclusions: Health interventions are required to increase parents' knowledge about photoprotection, in Mexico it shows that an educational intervention can increase the knowledge of young people in skin care in the future.
\end{abstract}

Keywords: Photoprotection, photoprotection in children, effects of radiation. 


\section{INTRODUCCIÓN:}

La piel es un órgano constituido por varios estratos, principalmente por los queratinocitos en un $90 \%$ y los melanocitos en $5-10 \%$, encargados de la formación de melanina $(1,2)$. La piel infantil presenta diferencias en comparación a la piel del adulto, tal como la capacidad disminuida de producir sudor, estratos más delgados, mayor permeabilidad y manto hidrolipídico escaso y menos resistente que genera una capacidad protectora menor, síntesis de melanina deficitaria, una superficie corporal mayor y un sistema inmunológico en vías de desarrollo $(1,2)$.

La piel infantil presenta una pobre función sudoral con tendencia a la resequedad, debido a que el manto hidrolipídico es menos resistente en comparación al del adulto a su vez es más delgada y permeable, que implica cuidados específicos, haciéndola susceptible a los daños producidos por la radiación solar $(1,2)$. La producción de melanina es menor en la piel del lactante y el preescolar por lo que fácilmente se puede producir quemaduras solares a pesar de una corta exposición $(1,2)$.

La exposición precoz a los rayos ultravioleta durante la infancia genera cambios degenerativos, carcinogénicos acumulativos e irreversibles (3). Las medidas de fotoprotección más comunes son el uso de barreras (sombrero de ala ancha, lentes y sombrillas) y el bloqueador solar. Se estima que un $80 \%$ de todos los canceres de piel pueden prevenirse adoptando medidas de fotoprotección. (3)

Poco se conoce sobre los conocimientos actitudes y prácticas de los padres hacia sus menores en relación a la fotoprotección en america latina. Por ello, el objetivo del artículo es identificar el nivel de conocimientos, actitudes y prácticas de los padres de países latinoamericanos sobre fotoprotección en menores de 14 años a través de la identificación de la evidencia científica publicada.

\section{MATERIALES Y MÉTODOS}

Estudio bibliométrico descriptivo, transversal y correlacional, realizado mediante búsqueda bibliográfica en Scopus, PubMed, y la Biblioteca Virtual de Salud con las palabras claves: "fotoprotección", "niños", "escolares" y sus equivalentes en inglés. La búsuqeda se realizó desde el uno de enero al 30 de junio de 2018. No hubo exclusión por idioma. Únicamente se incluyeron artículos originales.

\section{RESULTADOS:}

Se encontró un total de seis artículos, siendo dos de ellos realizados en población mexicana, y los otros en población uruguaya, cubana, peruana y chilena, independientemente.

En México, el $28.3 \%$ de los padres reportaron que sus hijos se exponen más de 60 minutos al sol entre semana; en el fin de semana se incrementa a un $42.8 \%$. El 23.2\% de los padres no utiliza alguna medida de fotoprotección y el $10 \%$ no utiliza bloqueador, así mismo el $99 \%$ de los padres saben que la exposición al sol es una causa de cáncer de piel, el $86.3 \%$ considera mortal el cáncer de piel y el $95 \%$ considera que el sol envejece (4). En otro estudio mexicano el $75.6 \%$ de los encuestados reconocieron saber los efectos de la radiación solar en la piel, el $86.3 \%$ sabe que el cáncer de piel puede ser mortal, sin embargo, a pesar de conocer los efectos nocivos el $32,4 \%$ considera beneficioso el bronceado. El $70.8 \%$ reconoció que utilizó de manera ocasional el protector solar (5).

En Uruguay el $62 \%$ de los niños encuestados era provenientes de familias de bajo nivel económico y educacional y el $32 \%$ a un nivel medio. El $42 \%$ indicaron que les gusta estar bronceados, el $19 \%$ se expuso al sol solo para broncearse. El $55 \%$ de los padres afirmó que se protegen del sol, el $30 \%$ solo a veces y el $15 \%$ no se protege; la falta de interés, el olvido y razones económicas son las causas por las que el $45 \%$ no se protegió. La medida de fotoprotección más utilizada fue la crema bloqueadora, seguida de los lentes y el uso rutinario de sombrero. La información acerca de la radiación solar y las medidas de fotoprotección fue proporcionada en un $75 \%$ por los medios de comunicación, de los médicos un $42 \%$ y el $5 \%$ no recibió información (6).

En un estudio chileno el $99.5 \%$ de los padres eran conscientes de la relación entre exposición solar y cáncer cutáneo, y el $84.2 \%$ consideraba peligroso que los niños se pongan "rojos"; la exposición al sol en verano ascendía al $59.6 \%$ al menos dos horas al día. El uso de protectores solares y gorros fueron las medidas más frecuentes $(85.4 \%$ y $826 \%$ respectivamente). Del total, niños de 6 meses y 2 años usaron gorro en un $87.2 \%$, el cuál disminuye con la edad, seguido del uso del filtro solar con $80.5 \%$ y la búsqueda de sombra con $79.2 \%$; Niños de 2 - 5 años usaron filtro solar en el $93.2 \%$ seguido del uso de gorro con $85.5 \%$. Finalmente, niños de 5 - 11 años usaron filtro solar con $84.1 \%$, el uso del gorro de un $76.6 \%$ y la búsqueda de sombra de un $62.3 \%$ (7).

El estudio realizado en Cuba el $72.5 \%$ han presentado al menos una quemadura solar, el $65.6 \%$ afirma usar cremas de protección solar. El empleo de bloqueador es más frecuente en las mujeres (77.4\%) que en los hombres (54.8\%). El 95.9\% no lo aplicaban en su hogar, el $94.7 \%$ no lo usaban todos los días y el $82.4 \%$ no lo replicaban en tres o más veces por día (8)

El estudio peruano demostró que el $48 \%$ utiliza protector solar en crema, el $46 \%$ ropa de protección y el $51 \%$ gorro - sombrero, la mayor fuente de información era conseguida a través de internet (59.4\%) seguida por el ministerio de trabajo (24.4\%).(9) 


\section{DISCUSIÓN:}

Las características propias de la piel en las primeras decadas de vida condicionan una mayor susceptibilidad ante el daño producida por la exposición prolongada a la radiación solar, siendo necesario el estudio del nivel de conocimiento de los padres sobre footoprotección de sus hijos. En general, se encontró un nivel adecuado de de conocimientos sobre fotoproteccion. Sin embargo, esto no se condice con las prácticas de los padres sobre fotoprotección en sus menores de edad.

Los menores son más susceptible a los efectos nocivos de la radiación ultravioleta, que largo plazo produce lesiones en el ácido desoxirribonucleico que promueve la formación de neoplasias cutáneas. 21 Existen tres de tipos de radiación ultravioleta, siendo la UVB la que se absorbe en un $90 \%$ por la epidermis y es la principal causante de las quemaduras solares, inmunosupresión y fotocarcinogénesis. (10 -12)

En los diversos artículos la mayoría de la población utiliza la crema con filtro solar como principal medida de fotoprotección, seguido del uso del gorro y la búsqueda de sombra, tanto en el estudio mexicano como chileno los padres tienen conocimiento de sobre la relación del cáncer de piel con la exposición al sol, en contraste con actitudes positivas sobre el bronceado. $(4,5,7)$ El estudio chileno presentó cifras similares en las horas de exposición de los niños al sol, siendo esta mayor de 2 horas asociado a menor uso de medidas de fotoprotección a mayor edad, siendo este fenómeno explicado por el menor control de los padres sobre los hijos en el cuidado de la piel. (7) En los estudios realizados en México, Uruguay y Chile no se hallaron mayores diferencias en cuanto al género en contraste con el realizado en Cuba donde las mujeres presentaban mayor uso de crema con filtro solar $(77.4 \%$ vs $55.8 \%)$. (4-7)

Dentro de las limitaciones del estudio, es la diversidad de las edades, y la falta de estudios limitados a la edad infantil. Sin embargo, este estudio sería una primer aproximación a un problema de interés actual.

\section{CONCLUSIÓN:}

La mayoría de estudios concluyen que los padres pueden tener conocimientos acerca de fotoprotección y su asociación al cáncer de piel, pero con actitudes contradictorias dejando de lado la protección de los niños frente a los efectos de la radiación solar. En los estudios se evidencia el antecedente de quemadura solar en casi la mitad de la población que indica exposición prolongada la radiación solar. La edad infantil constituye un grupo prioritario para las intervenciones sanitarias a fin de disminuir la exposición precoz y excesiva a la radiación y disminuir las complicaciones a corto y largo plaza producida por esta. Las intervenciones educativas en la población infantil constituyen una gran medida para empoderar a la población sobre fotoprotección. 


\section{REFERENCIAS BIBLIOGRÁFICAS}

1. Gilaberte Y. y Carrascosa J.M. Realidades y retos de la fotoprotección en la infancia. Actas Demosifiliorg. 2014.; 105(3):253-62

2. Lansen R, Q Wang S. Burnett M, Osterwalder U, Lim H. Photoprotection. En: JAAD.2013;6,(69):1-12

3. Ramírez E, Ferrán M, Torres D, Suarez L, Lima R. Protección Solar en niños. Rev Méd Electrón. 2016; 38(2):177-84. Disponible en: http://www.revmedicaelectronica.sld.cu/index.php/rm e/article/view/2946/1714

4. Cos-Reyes A. Chico-Barba Gabriela, Ferreira, Jaime F. Conocimientos y actitudes parentales sobre efectos de la exposición solar y fotoprotección en niños. Rev Mex. Pediatr. 2016;83 (3):74-79

5. Vera $S$, Gonzales $P$ y Domínguez J. Intervención educativa en los conocimientos, actitudes y prácticas sobre la protección solar en estudiantes de educación secundaria. DermatologíaCMQ.2013;11(2):94-103. Disponible http://www.medigraphic.com/pdfs/cosmetica/dcm-2 013/dcm132d.pdf

6. Magliano J, Bálsamo A, Ruibal F, Alvarez M, Bazzano C. Hábitos de fotoprotección en los niños que concurren a Dermatología pediátrica del Centro hospitalario Pereira Rossell. Arch Pediatr Urug. 2016. 87(3):210 20.
7. Castillon M. A. Matamala J. Feldman M, Gajazdo C, Valenzuela C y Zemelman V. Prácticas de exposición solar en padres de niños en Atención Primaria, Santiago Chile. Rev Hosp Clín Univ Chile 2013; 24: 6 $-13$

8. Alemán Bacallao AD, Guerra Castro MM. Conocimientos, hábitos y actitudes de fotoprotección en adolescentes. Rev Méd 2018. 40(3). 3-11.

9. Llasaca Calisaya E. Relación entre el nivel de conocimiento y las prácticas de fotoprotección sobre los efectos de la radiación solar en la población del distrito de Moquegua. UNJBG. Tacna. 2017

10. American Cancer Society. ¿Qué es la Radiación Ultravioleta? 2017 [fecha de acceso 10 de junio 2018) URL disponible en https://www.cancer.org/es/cancer/cancer-de-piel/pr evencion-y-deteccion-temprana/que-es-la-radiacio n-de-luz-ultravioleta.html

11. Barco Nebreda D, Gonzales Quarante L. Dermatología. Primera Edición. CTO Editorial México. 2013

12. ${ }^{\circ}$ Cardona - Hernández MA, Fierro - Arias L. Cabrera Perez AL. Vidal - Flores AA. Efectos de la Radiación Electromagnética en la Piel. Dermatol Rev Mex 2017.61(4):292-302.

\section{Las ediciones anteriores de revista médica PANACEA están disponibles en:}

\section{www.revpanacea.unica.edu.pe}

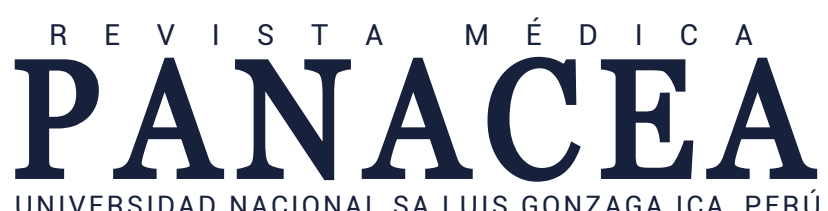

UNIVERSIDAD NACIONAL SA LUIS GONZAGA ICA, PERÚ 